\title{
RESTRICCIONES AL DERECHO DE ACCESO A LA INFORMACIÓN PÚBLICA EN LAS RESOLUCIONES DE LOS ÓRGANOS GARANTES LOCALES \\ - $\mathrm{O}$
}

José Guadalupe LUNA HERNÁNDEZ*

\section{Planteamiento}

Desde julio de 2015 a la fecha, ${ }^{1}$ el Pleno del Instituto de Acceso a la Información Pública y Protección de Datos Personales del Estado de México y Municipios ha resuelto 31 recursos de revisión interpuestos por diversas personas en contra de las respuestas que les fueron notificadas por las autoridades a sus solicitudes de acceso a la información pública. Particularmente, las solicitudes versan sobre la licitación de obras de alto impacto, entre ellas el Viaducto Bicentenario, el Museo Torres Bicentenario y el Circuito Exterior Mexiquense. En estos casos, las autoridades han negado el acceso a la información solicitada porque existen diversos procedimientos judiciales y administrativos en curso, y con fundamento en una cuestionada interpretación de la fracción VI del artículo 20 de la ley estatal de transparencia se reserva la información al tratarse de procedimientos que no han causado estado.

Los solicitantes, en consecuencia, han interpuesto el recurso de revisión ante el órgano garante tratando de demostrar que la respuesta limita su derecho. En dos recursos, el 01435/INFOEM/IP/RR/2015 y el 1444/ INFOEM/IP/RR/2015, y los acumulados de ambos, la autoridad pretendió clasificar como información reservada la de diversos actos previos al otorgamiento de la concesión empleando un acuerdo de clasificación que

* Licenciado en ciencia política y administración pública por la Facultad de Ciencias Políticas y Sociales de la UNAM; comisionado del Instituto de Transparencia y Acceso a la Información Pública del Estado de México y Municipios, jose.luna@itaipem.org.mx.

Fecha de recepción: 5 de enero de 2016.

Fecha de dictamen: 7 de marzo de 2016.

1 El estudio fija como inicio temporal el mes de julio de 2015 porque a partir del día siete de ese mes fui designado como comisionado en el órgano garante del Estado de México. 
sólo incluía al contrato y los actos posteriores, y señalando sólo las causas judiciales en los que se impugnaron éstos.

Son esas resoluciones a los recursos de revisión mencionados a las que a continuación me referiré, ya que si bien, aunque no correcto, los sujetos obligados pueden intentar restringir el acceso a la información, lo que resulta desafortunado es que sea el órgano garante estatal quien sistemáticamente confirme las respuestas o las modifica para efectos de que se emitan los acuerdos de clasificación o se corrijan ante las evidentes deficiencias que demuestran. La tendencia de dicho órgano preserva la decisión inicial de impedir el acceso a la información solicitada, lo que implica una clara - declinación de su naturaleza de órgano de control y tutela del derecho en - cuestión. Para justificar su actuar, se observa en estas resoluciones una indebida interpretación de la norma jurídica antes señalada. En el desarrollo del presente comentario mostraré por qué esa interpretación es del todo inadecuada e identificaré los graves efectos que provoca y que constituyen una evidente afectación al derecho de acceso a la información pública.

\section{LOS RECIENTES RECURSOS DE REVISIÓN}

En el caso de los recursos de revisión 01435/INFOEM/IP/RR/2015 y 1444/INFOEM/IP/RR/2015, y los acumulados de ambos, ${ }^{2}$ las respuestas a las solicitudes de acceso a la información y los argumentos vertidos en los informes de justificación se sustentaron en un acuerdo del Comité de Información del Sistema de Autopistas, Aeropuertos, Servicios Conexos y Auxiliares del Estado de México (SAASCAEM), que clasifica como información reservada “...el Título de Concesión del Sistema Carretero del Oriente del Estado de México (Circuito Exterior Mexiquense), incluyendo todas sus modificaciones y anexos, así como la información referente a obras adicionales relativas al monumento bicentenario...". Dicha respuesta fue del todo inadecuada, ya que a través de las solicitudes se pretende acceder a otro tipo de información no comprendida en dicho acuerdo, consistiendo dicha información en:

- Copia de las Bases de Licitación del concurso SCEM-CCA-01-02 para la adjudicación de la concesión para la construcción, explotación, operación, conservación y mantenimiento del Sistema Ca-

2 Ambas resoluciones con sus acumulados fueron aprobadas por mayoría, con voto disidente del comisionado José Guadalupe Luna Hernández, la primera el 6 y la segunda el 13 de octubre de 2015. 
rretero del Oriente del Estado de México (Circuito Exterior Mexiquense); así como todas sus modificaciones, apéndices y/o anexos correspondientes.

- Copia de todas las actas de las juntas de aclaraciones celebradas en relación con el referido concurso SCEM-CCA-01-02.

- Copia de todas las preguntas formuladas por los participantes de dicho concurso y de las respuestas a cada una de dichas preguntas.

Ante ello, y considerando que dicha información no estaba comprendida en el acuerdo de clasificación señalado previamente y que tampoco era materia de los procedimientos jurisdiccionales que se desahogaban, luego de la interposición de los recursos de revisión - en el informe de justificación - , las autoridades aportaron información adicional para tratar de conservar la clasificación realizada: los datos de un juicio administrativo que involucra actos distintos a los combatidos en los amparos y que consisten en la información a la que pretenden acceder las personas en estos casos. De esta forma, la restricción se funda en dos aspectos: $a$ ) una determinación unilateral y defectuosa de origen, ya que para tal efecto no se emitió en su momento un nuevo acuerdo de clasificación, y $b$ ) en una interpretación equivocada de la fracción VI del artículo 20 de la ley estatal de transparencia, auspiciada desde el propio órgano garante. Esta segunda cuestión es la que se comenta a continuación.

\section{El CRITERIO RESTRICTIVO EN LA INTERPRETACión}

Si los sujetos obligados aducen como argumento para tratar de clasificar como información reservada aquella que se encuentra inmersa en cualquier tipo de procedimiento, es porque ha sido una práctica constante de la mayoría de los integrantes del órgano garante el señalar que cuando la información se relacione con cualquier procedimiento administrativo, judicial o una investigación, si aún no ha causado estado, debe clasificarse como reservada. Tal decisión es una interpretación mutativa de carácter reductor del precepto jurídico (Díaz, 2011: 132), ya que elimina, para efectos de cada caso en resolución; la parte más significativa señalada en la fracción VI del artículo 20 de la ley estatal de transparencia que a la letra dice:

Artículo 20. Para los efectos de esta Ley, se considera información reservada, la clasificada como tal, de manera temporal, mediante acuerdo fundado y motivado, por los sujetos obligados cuando: ...VI. Pueda causar daño o 
alterar el proceso de investigación en averiguaciones previas, procesos judiciales, procesos o procedimientos administrativos, incluidos los de quejas, denuncias, inconformidades, responsabilidades administrativas y resarcitorias en tanto no hayan causado estado;

La sana interpretación de la norma en cuestión consiste en señalar que cuando se trate de información relacionada con un procedimiento ad74 ministrativo, judicial o una investigación, y que no hayan causado estado, se deberá entregar la información si ésta no puede causar daño o alterar el desarrollo del procedimiento. La interpretación que se ha impuesto en

- este tipo de resoluciones provoca el incumplimiento de un mandato expre- so de la ley de transparencia local, la que además aún no se ha armonizado, por lo que omite lo que la Ley General de Transparencia denomina como la "prueba de daño". ${ }^{3}$ En el caso en cuestión, si bien es cierto que el sujeto obligado cada vez precisa con mayores elementos los procedimientos jurisdiccionales en curso y señala los agravios que constituyen la materia de estos procesos, también lo es que de haberse realizado un adecuado razonamiento lógico jurídico, el sujeto obligado tendría que haber aportado elementos adecuados para generarle al órgano garante una convicción plena de que la entrega de la información en cuestión podría generar un daño al desarrollo de la investigación, y no la manifestación de fe a la que terminan restringidas las resoluciones.

\section{LA PRUEBA DE DAÑO}

No basta con que exista un procedimiento para clasificar la información como reservada; se requiere apreciar objetiva, clara e indubitablemente que entregar la información puede afectar el proceso en su conjunto, ya sea por la fase en la que se encuentra el procedimiento o por su contenido. El hecho de clasificar como información reservada convocatorias de licitación, títulos de concesión o contratos que en su momento fueron publicados incluso en la Gaceta de Gobierno del Estado, o que aún se encuentran disponibles en Internet, en sitios electrónicos oficiales o no, no sólo es un intento infructuoso, sino que a veces provoca que las decisiones gubernamentales se aparten del sentido común. Clasificar como información reservada documentos ubicados en las primeras fases del proceso de investigación iniciados y que podrían mostrar si está siendo exhaustivo, deficiente o incompleto, en los casos en los que se reconoce la acción po-

3 Diario Oficial de la Federación, 4 de mayo de 2015. 
pular, podría contribuir a que quien cuente con información que pueda ser útil para la investigación se acerque a la autoridad y le proporcione medios que podrían fortalecer la acción investigadora de la autoridad, lo que lejos de afectarla puede justificar claramente la entrega de la información que se ha reservado.

Aun sin armonizar, las disposiciones jurídicas existentes en nuestra entidad precisan la necesidad de que se acredite el probable daño presente, probable y específico, lo que se contempla en los "Lineamientos para la recepción, trámite y resolución de las solicitudes de acceso a la información pública, acceso, modificación, sustitución, rectificación o supresión parcial o total de datos personales, así como de los recursos de revisión que deberán observar los sujetos obligados por la Ley de Transparencia y Acceso a la Información Pública del Estado de México y Municipios" (numerales cuarenta y seis y cuarenta y siete). Para que operen las restricciones de acceso a la información en poder de los sujetos obligados se exige actualizar los supuestos normativos aplicables a cada caso, lo que implica la emisión del acuerdo del Comité de Información que clasifique la información (artículo 21 de la ley estatal), que en algunos casos no existía al momento de emitir la resolución, y que además debe cumplir con lo siguiente:

I) Un razonamiento lógico que demuestre que la información encuadra en alguna de las hipótesis de excepción previstas en la Ley (fundamentación y motivación).

II) Que la liberación de la información de referencia pueda amenazar efectivamente el interés protegido por la Ley (existencia de intereses jurídicos).

III) La existencia de elementos objetivos que permitan determinar si la difusión de la información causaría un daño presente, probable y específico, a los intereses jurídicos tutelados en los supuestos de excepción previstos en la Ley (elementos de la prueba del daño).

En el entendido de que dichos extremos legales tienen el siguiente alcance: por daño presente se entiende que de publicarse la información a la fecha en que se realiza el análisis correspondiente, se generará la afectación respectiva a cualquiera de los valores o bienes jurídicos tutelados en los casos de excepción previstos en los artículos 20 y 24 de la Ley de Transparencia; por daño probable se establece que la difusión de la información en cuestión pueda causar un perjuicio mayor al interés público de conocer la información; por daño específico, el que inmediatamente después de la publicación de la información sea inminente la materialización o afectación a los intereses jurídicos tutelados en los supuestos de excepción. 
En los casos resueltos, la reserva que bajo algún criterio extremo pudiera ser correcta termina siendo inválida porque no se observaron las formalidades que se han señalado y que son indispensables e insustituibles para acreditar fehacientemente las condiciones de excepcionalidad que caracterizan al procedimiento de clasificación de la información como reservada.

La mayoría del órgano garante consideró que en una propuesta económica que haya sido ganadora de un proceso de licitación, resulta razonablemente lógico pensar que quienes tienen un interés directo en los procedimientos judiciales y administrativos son las personas o empresas que participaron en el proceso de licitación. Si esto fuera así, en nada afectaría la entrega de la bases de licitación si precisamente quienes participaron en el proceso de licitación debieron de haberlas adquirido, como señalan las bases I, II y XII de la Convocatoria a la licitación pública núm. SCEM-CCA-01-02, publicada en la Gaceta del Gobierno del 28 de octubre de 2002. En consecuencia, los procedimientos no se verían afectados; cualquiera de las posibles partes de los procedimientos judiciales no obtendrían una ventaja indebida si para participar en el proceso de licitación debieron adquirir las bases que son los documentos a los que pretenden acceder los solicitantes. En este caso, resulta evidente que la afectación al proceso jurisdiccional es inexistente.

En cuanto a las modificaciones, apéndices y/o anexos correspondientes de esas mismas bases, es necesario señalar que el artículo 35, fracción VI, de la Ley de Contratación Pública del Estado de México y Municipios establece que éstas se harán del conocimiento de los interesados hasta tres días hábiles antes de la fecha señalada para el acto de presentación y apertura de propuestas. En consecuencia, es razonablemente lógico que dicha información haya estado disponible para todas aquellas personas o empresas que participaron en el proceso de licitación, por lo que si partimos de que las posibles partes de los procesos jurisdiccionales son esas mismas personas, resulta evidente que la entrega de dicha información en nada puede afectar el proceso, ya que es pública, incluso desde antes del otorgamiento de la concesión.

Por lo que respecta a las actas de las juntas de aclaraciones, debe señalarse que según lo dispuesto por el artículo 77 del reglamento de la ley, referida en el párrafo anterior, estos documentos estuvieron disponibles para los participantes de la licitación en el domicilio que haya señalado la convocante o en Compramex. Por lo tanto, es evidente que en nada dañaría la entrega de esta información, que previo al procedimiento jurisdiccional o administrativo es susceptible que estuviera a disposición y en posesión de las partes del mismo. 
Esto es igual en cuanto hace a las preguntas y respuestas formuladas en dichas juntas, ya que el artículo 79, fracción III, del reglamento señalado establece que el acta de las juntas de aclaraciones debe contener las preguntas y las aclaraciones respectivas y si, como se ha señalado antes, las posibles partes del procedimiento han podido acceder a dicha información, entonces en nada afectaría a la igualdad de armas o equidad del proceso el que se permita el acceso a la información.

Debe señalarse que la limitada información que proporciona la autoridad sobre el seguimiento del procedimiento no nos permite apreciar en qué situación se encuentra para valorar si la fase probatoria ha sido concluida, lo que permitiría advertir el grado de posible afectación que generaría la entrega de la información requerida. Si lo anterior no fuera poco, el órgano garante se ha negado a practicar una prueba adicional que debería contrastarse con la prueba de daño: el interés público.

\section{El DAÑo FRENTE AL INTERÉS PÚBLICO}

Cuando una persona acude al procedimiento de solicitud de acceso a la información pública es evidente que lo hace porque se ve en la necesidad de conocer la actuación de los agentes gubernamentales, ya sea porque le genera duda, inconformidad o molestia la forma en como han actuado las instituciones (Merino, 2008: 243). Con raras excepciones puede considerarse que se pretende acceder a la información por el apoyo que le provoca la decisión gubernamental. El derecho de acceso a la información, como otros derechos, es parte de la esfera de lo indecidible, aquello que no sólo legitima, sino que incluso deslegitima las decisiones y las no decisiones, por lo que pretende ser un límite a la actuación gubernamental (Ferrajoli, 2010: 24). Por lo tanto, no sería ilógico que lo que pretendan los solicitantes sea conocer lo que ha ocurrido en esta materia, formarse una opinión informada y ejercer cualquier otro derecho, ya sea el de libertad de expresión o los derechos políticos que le reconocen acción popular para presentar denuncias en contra de cualquier funcionario público que haya cometido alguna infracción (Schamis, 2009: 72).

Por eso es que en estos recursos de revisión, y para analizar la pretendida reserva de la información que en algunos casos incumple con las formalidades que se exigen para que sea considerada válida, resulta indispensable realizar la ponderación entre el daño que puede provocar su difusión y el interés público de que se dé a conocer, lo que señala el artículo 146 de la Ley General de Transparencia y Acceso a la Información Pública. Esta 
JOSÉ GUADALUPE LUNA HERNÁNDEZ

prueba, además, encuentra sustento jurisdiccional en la tesis de jurisprudencia que precisa:

INFORMACIÓN RESERVADA. EXCEPCIÓN A LA PROHIBICIÓN DE SU DIVULGACIÓN. En términos de las fracciones IV y VI del artículo 14 de la Ley Federal de Transparencia y Acceso a la Información Pública Gubernamental, tanto los expedientes judiciales que no hayan causado estado, como las opiniones, recomendaciones o puntos de vista que formen parte del proceso deliberativo de los servidores públicos, mientras no sea adoptada la decisión definitiva, constituyen información reservada. No obstante la imposibilidad de acceder a dicha información no puede considerarse como una regla absoluta, porque en aquellos supuestos en los cuales su difusión producirá mayores beneficios para la sociedad que los daños que pudieran provocarse con su divulgación, debe hacerse una excepción a la regla general, privilegiando la transparencia y difusión de la información respectiva (Tesis P./J. 45/2007).

Para valorar si en los procedimientos que se resuelven mediante estas resoluciones existía un interés público mayor por privilegiar la transparencia y difundir la información solicitada que derrote la clasificación de la información como reservada, resultaba necesario considerar los siguientes aspectos: la convocatoria para la respectiva licitación ${ }^{4}$ requirió que los participantes acreditaran un capital contable mínimo de 400 millones de pesos. Según el documento "BVA Bancomer financiará y estructurará el sistema carretero del Oriente del Estado de México" (BBVA: 2004), disponible en Internet, de 6 de diciembre de 2004, la "longitud total será de 142 kilómetros con un costo aproximado de $\$ 6,628$ millones de pesos y que permitirá comunicar las autopistas México-Querétaro, México-Pachuca, México-Puebla, y los límites del Estado de Morelos”.

Según ese mismo documento, que se encuentra disponible en Internet, el gobierno del Estado de México otorgó la concesión a la empresa constructora española Obrascón Huarte Laín S. A. (OHL), la cual constituyó en México la empresa Concesionaria Mexiquense S. A. de C. V. (Conmex), y vale la pena transcribir una parte más del citado boletín: "Con estas obras se tiene programado satisfacer una demanda de tráfico de 10,000 vehículos diarios en promedio, beneficiando a los usuarios de los municipios del Estado de México de Coyotepec, Zumpango, Nextlalpan, Coacalco, Ecatepec y Atenco, e indirectamente a las delegaciones Azcapotzalco y Miguel Hidalgo". Adicionalmente, es de pleno y notorio conocimiento público que la filtración de llamadas telefónicas en las que

4 Publicada en la Gaceta de Gobierno del Estado el 28 de octubre de 2002, y a la que se podía acceder sin ninguna restricción vía Internet hasta octubre de 2015 (GEM: 2002). 
participaron el anterior secretario de la extinta Secretaría de Comunicaciones del Gobierno del Estado de México y funcionarios de la empresa OHL provocaron no sólo la salida del ex funcionario, sino también el inicio de procedimientos de investigación por sospechas de corrupción.

De lo hasta aquí señalado puede apreciarse que la información requerida por los solicitantes no consistió en la pavimentación de una calle cualquiera del territorio estatal, sino de una obra de infraestructura que involucra cuantiosos recursos económicos. El presupuesto total de la obra, según la información dada a conocer por Bancomer, representaría el $34.53 \%$ del total de los recursos ejercidos en 2014 por el sector central del gobierno del estado en el capítulo "6,000 Inversión pública" (GEM, 2015). Asimismo, la obra afecta a una región sensible del estado y a una parte significativa de la población de esta entidad y de las colindantes, así como de la capital de la República. Se trata de una concesión y operación sobre la que pesan sospechas de actos de corrupción que han alcanzado a por lo menos un mando superior de la administración pública del estado y que atrae la atención pública nacional e internacional al respecto. En razón de lo anterior, es dable valorar entonces el interés público de que las personas accedan a la información y que este interés público sea suficiente para restringir la necesidad de clasificar la información como reservada.

Según lo que dispone la fracción XII del artículo tercero de la Ley General de Transparencia y Acceso a la Información Pública, por información de interés público debemos entender aquella "información que resulta relevante o beneficiosa para la sociedad y no simplemente de interés individual, cuya divulgación resulta útil para que el público comprenda las actividades que llevan a cabo los sujetos obligados". Frente a esa definición, el órgano garante debió reflexionar si el acceso a la información tenía un mero interés individual o si, dada la atención que han generado estos acontecimientos, entregarla resultaba beneficioso para la sociedad. Desde mi punto de vista, esto es así porque permitir el acceso a la información solicitada es la medida idónea, necesaria y proporcional que propicia un beneficio mayor al aplicar el principio de máxima publicidad sobre la condición excepcional de la reserva de la información.

El órgano garante debió considerar lo dispuesto por el artículo 101, fracción III, de la citada Ley General que señala que "Los documentos clasificados como reservados serán públicos cuando... (e)xista resolución de una autoridad competente que determine que existe una causa de interés público que prevalece sobre la reserva de la información”; en el caso en cuestión, si bien algunos de los documentos ya habían sido clasificados como reservados y otros no, toda vez que no se emitió el acuerdo respecti- 
vo, en consecuencia, sólo se había impedido el acceso a los mismos. Incluso, era dable que mediante la resolución del órgano garante se determinara que existía una clara "causa de interés público" en estos casos, y que no es otra distinta a que la sociedad conozca los términos del proceso para la licitación de la concesión, y con ello se genere una opinión informada que le permita manifestarse en ejercicio de su derecho de libertad de expresión. Además, era deseable que de los propios documentos a los que acceda un ciudadano, y si cuenta con información adicional, se aporten elementos adicionales a la autoridad encargada de realizar la investigación para asegurar un mejor ejercicio del procedimiento respectivo. Por ello, lejos de

- impedir el acceso a la información, debería reconocerse la prudencia de las - personas solicitantes de la información que proceden pretendiendo acceder a la información necesaria para participar en la arena pública.

En estos casos, resulta claro el interés público para que las acciones gubernamentales sean sujetas a un escrutinio público, favoreciendo la transparencia y la debida rendición de cuentas, como lo recomiendan diversos autores (Aguilar, 2008: 29), mucho más cuando sobre dichos actos pesan indicios graves de corrupción que deben ser investigados. El objetivo es que dicha investigación sea pronta, expedita y, lo más importante, sujeta a control popular (Moreno, 2014: 347), como un mecanismo esencial para no permitir que prevalezca la discrecionalidad y la secrecía que caracterizó a las fases iniciales del proceso y que provocaron los presumibles actos de corrupción que hoy se investigan. Por ello, estoy convencido de que bajo el supuesto antes señalado de la Ley General de Transparencia y Acceso a la Información Pública y los criterios jurisdiccionales citados en la presente sección resultaba más que razonable ordenar la entrega de la información requerida.

\section{LA INTERPRETACIÓN MUTATIVA Y SUS CONSECUENCIAS}

Como he señalado en diversas opiniones particulares, ${ }^{5}$ y posteriormente en votos disidentes, ${ }^{6}$ es recurso legítimo del operador jurídico la interpretación mutativa de las disposiciones. Dicho método de interpretación conforme siempre tiene como finalidad preservar bienes constitucionalmente

5 Opinión particular emitida por el comisionado José Guadalupe Luna Hernández en las resoluciones 01015/INFOEM/IP/RR/2015, 01017/INFOEM/IP/RR/2015, 01435/INFOEM/IP/RR/2015 y en sus acumulados.

6 En las resoluciones 01435/INFOEM/IP/RR/2015 y 1444/INFOEM/IP/RR/2015 con sus acumulados. 
señalados o derechos humanos, evitando la expulsión de la literalidad de la norma secundaria del ámbito jurídico a partir de la mutación que se hace a su significado. En el caso en cuestión, nos encontramos frente a actos de autoridad sobre los cuales pesan notorias sospechas por la probable comisión de actos de corrupción. Bajo ese contexto, las personas han mostrado un creciente interés por conocer de primera mano la información relacionada con el caso, por lo que pretender que una interpretación que impida a la opinión pública el acceso a los documentos no abona al fortalecimiento de la cultura de la rendición de cuentas ${ }^{7}$ ni prestigia ni legitima a la autoridad. Por el contrario, lesiona gravemente el modelo constitucional de transparencia, del sistema de rendición de cuentas y el derecho humano de acceso a la información. Así que siendo los efectos contrarios a principios constitucionales y derechos humanos, una interpretación restrictica no sólo debe ser desestimada, sino combatida por ser derecho ilegítimo (Ferrajoli, 2014: 72).

Pero con la negativa sistemática del órgano garante a practicar la prueba de daño y confrontar su resultado con el interés público de que dicha información se abra, y aprovechándose de la falta de armonización de la ley estatal, se asume esta interpretación mutativa de carácter restrictivo. La principal consecuencia que puede apreciarse de esta indebida interpretación y esa deliberada decisión de no practicar la prueba de daño ni confrontar el interés público consiste en que el procedimiento de investigación, lejos de contribuir a la cultura de la transparencia, se constituye como un nuevo obstáculo, el cual puede ser administrado y manipulado por la autoridad a la que se le pretende exigir cuentas. De esta forma, sus resultados y efectos se diluyen por el paso del tiempo u ocurren en un momento en el que los actos repudiados son irreversibles o la sanción es de nula trascendencia para la sociedad. Con eso sólo pierde el Estado de derecho, el Estado constitucional de derecho y la sociedad democrática.

\section{BibLiografía}

Aguilar Rivera, José Antonio, 2008, Transparencia y democracia: claves para un concierto, México, IFAI, 4a. ed., Cuadernos de Transparencia, núm. 10.

7 Principio establecido desde la Declaración de los Derechos del Hombre y del Ciudadano de 1789, que en el artículo 15 señalaba: "La sociedad tiene derecho a pedir cuentas de su gestión a todo agente público". 
Esta revista forma parte del acervo de la Biblioteca Jurídica Virtual del Instituto de Investigaciones Jurídicas de la UNAM

JOSÉ GUADALUPE LUNA HERNÁNDEZ

BBVA BANCOMER, 2004, "BBVA Bancomer financiará y estructurará el sistema carretero del Oriente del Estado de México", Sala de Prensa, disponible en http://press.bbva.com/latest-contents/press-releases/bbva-bancomer -financiara-y-estructurara-el-sistema-carretero-del-oriente-del-estado-demexico_9882-22-c-1081_.html, consultado el 6 de diciembre de 2016.

Díaz Revorio, F. Javier, 2011, Las sentencias interpretativas del Tribunal Constitucional, México, Porrúa-Instituto Mexicano de Derecho Procesal Constitucional.

Ferrajoli, Luigi, 2010, Derechos y garantías. La ley del más débil, 7a. ed., Madrid, Trotta.

- - 2014, La democracia a través de los derechos. El constitucionalismo garantista como modelo teórico y como proyecto político, Madrid, Trotta.

Gobierno del Estado de México (GEM), 2015, Cuenta Pública del Gobierno y Organismos Auxiliares y Autónomos del Estado de México del Ejercicio Fiscal 2014. Resultados Generales, t. I.

_ 2002, "Licitación pública núm. SCEM-CCA-01-02", Periódico Oficial del Estado Libre y Soberano de México, Gaceta del Gobierno, disponible en http://legislacion.edomex.gob.mx/sites/legislacion.edomex.gob. $m x / f i l e s / f i l e s / p d f / g c t / 2002 / o c t 283 . p d f$, consultado el 12 de abril de 2016.

Merino, Mauricio, 2008, "La transparencia como política pública", en AcKeRman, John M. (coord.), Más allá del acceso a la información: transparencia, rendición de cuentas y Estado de derecho, México, UNAM-Siglo XXI Editores-Universidad de Guadalajara-Cámara de Diputados-Centro Internacional de Estudios sobre Transparencia e Información.

Moreno Espinosa, Roberto, 2014, "Del patrimonialismo a la transparencia en la administración pública de México. Un largo y sinuoso camino... y lo que nos falta recorrer", Comisión de Vigilancia de la Auditoría Superior de la Federación, Cámara de Diputados del H. Congreso DE La Unión, LXII Legislatura, Fiscalización, transparencia y rendición de cuentas, Naucalpan, CVASF de la CDHCU.

Resolución 01015/INFOEM/IP/RR/2015 y acumulados, Instituto de Transparencia, Acceso a la Información Pública y Protección de Datos Personales del Estado de México y Municipios.

Resolución 01017/INFOEM/IP/RR/2015 y acumulados, Instituto de Transparencia, Acceso a la Información Pública y Protección de Datos Personales del Estado de México y Municipios.

Resolución 01435/INFOEM/IP/RR/2015 y acumulados, Instituto de Transparencia, Acceso a la Información Pública y Protección de Datos Personales del Estado de México y Municipios, octubre de 2015. 
Resolución 1444/INFOEM/IP/RR/2015 y acumulados, Instituto de Transparencia, Acceso a la Información Pública y Protección de Datos Personales del Estado de México y Municipios, octubre de 2015.

Schamis, Héctor E., 2009, "Evitando la colusión, previniendo la colisión: ¿qué sabemos de la economía política de la privatización?”, en SANDovaL, Irma Eréndira (coord.), Corrupción y transparencia. Debatiendo las fronteras entre Estado, mercado y sociedad, México, UNAM-Siglo XXI Editores.

Tesis P./J. 45/2007, disponible en https://www.scjn.gob.mx/pleno/SecretariaGeneralDeAcuerdos1/TesisJurisprudencialesdelPleno/2007/40TJ45-2007. $p d f$. 